\title{
Evaluation of the stereochemical quality of predicted RNA 3D models in the RNA-Puzzles submissions
}

\author{
FRANCISCO CARRASCOZA, ${ }^{1,6}$ MACIEJ ANTCZAK, ${ }^{1,2,6} \mathrm{ZHICHAO} \mathrm{MIAO,,}^{3,4}$ ERIC WESTHOF, \\ and MARTA SZACHNIUK ${ }^{1,2}$ \\ ${ }^{1}$ Institute of Computing Science and European Centre for Bioinformatics and Genomics, Poznan University of Technology, 60-965 Poznan, Poland \\ ${ }^{2}$ Institute of Bioorganic Chemistry, Polish Academy of Sciences, 61-704 Poznan, Poland \\ ${ }^{3}$ European Molecular Biology Laboratory, European Bioinformatics Institute (EMBL-EBI), Wellcome Genome Campus, Hinxton CB10 1SD, \\ United Kingdom \\ ${ }^{4}$ Translational Research Institute of Brain and Brain-Like Intelligence, Department of Anesthesiology, Shanghai Fourth People's Hospital Affiliated \\ to Tongji University School of Medicine, Shanghai 200081, China \\ ${ }^{5}$ Université de Strasbourg, Institut de Biologie Moléculaire et Cellulaire CNRS, Architecture et Réactivité de I'ARN, 67084 Strasbourg, France
}

\begin{abstract}
In silico prediction is a well-established approach to derive a general shape of an RNA molecule based on its sequence or secondary structure. This paper reports an analysis of the stereochemical quality of the RNA three-dimensional models predicted using dedicated computer programs. The stereochemistry of 1052 RNA 3D structures, including 1030 models predicted by fully automated and human-guided approaches within 22 RNA-Puzzles challenges and reference structures, is analyzed. The evaluation is based on standards of RNA stereochemistry that the Protein Data Bank requires from deposited experimental structures. Deviations from standard bond lengths and angles, planarity, or chirality are quantified. A reduction in the number of such deviations should help in the improvement of RNA 3D structure modeling approaches.
\end{abstract}

Keywords: stereochemistry; quality validation; RNA structure; 3D structure prediction; RNA-Puzzles

\section{INTRODUCTION}

Knowledge of the RNA atomic structure is crucial to address biological problems, therefore computational tools for the prediction of RNA three-dimensional models from the sequence have been developed to help or bypass some hurdles of laboratory procedures (Lukasiak et al. 2015; Miao and Westhof 2017; Gumna et al. 2020; Li et al. 2020; Magnus et al. 2020).

The first decade of the 21 st century resulted in several computer programs and protocols, which paved the way for automated modeling of RNA 3D structures: S2S (Jossinet and Westhof 2005), FARFAR (Das and Baker 2007), iFoldRNA (Ding et al. 2008), MC-Fold/MC-Sym (Parisien and Major 2008), and NAST (Jonikas et al. 2009). Some of them developed into highly specialized programs, which are used for either fully automatic or human-guided prediction. In the following years, this collection grew to include other tools such as ModeRNA (Rother et al. 2011), RNAComposer (Popenda et al. 2012), 3dRNA (Zhao et al.

\footnotetext{
${ }^{6}$ Joint first authors.
}

Corresponding author: mszachniuk@cs.put.poznan.pl

Article is online at http://www.rnajournal.org/cgi/doi/10.1261/rna .078685 .121 . Freely available online through the RNA Open Access option.
2012), Vfold (Xu et al. 2014), and SimRNA (Boniecki et al. 2016).

To stimulate the improvement of quality in RNA prediction, RNA-Puzzles was organized $10 \mathrm{yr}$ ago (Cruz et al. 2012). RNA-Puzzles is a community-wide assessment of RNA 3D structure prediction that aims to understand the bottlenecks in current RNA 3D structure prediction to promote the improvement of prediction methods. Before the publication of an experimentally determined RNA structure, the sequence is disseminated among the community and prediction results are submitted within 3-4 wk. Assessment against the experimental structure is performed after the release of the structure. There are two categories of challenges, depending on the protocols used to obtain the models: They can originate from fully automated web services or human experts running various prediction programs. The starting point for each challenge is a novel experimentally determined RNA 3D structure, the conformation of which is unknown to the predictors. The web servers have $48 \mathrm{~h}$ and human experts 3-4 wk for

(C) 2022 Carrascoza et al. This article, published in RNA, is available under a Creative Commons License (Attribution-NonCommercial 4.0 International), as described at http://creativecommons.org/licenses/ by-nc/4.0\%. 
submitting their models. After the deadline, the predictions are evaluated and the results are published with the ranking of the submitted models. Presently, 28 crystallographic structures have been part of the contest. Eighteen of them have been the basis of four scientific papers published by the RNA-Puzzles community (Cruz et al. 2012; Miao et al. 2015, 2017, 2020). As of October 2020, 22 challenges have been concluded with assessment results available on the RNA-Puzzles website (http ://www.rnapuzzles.org). It provides accuracy assessments determined in comparison with the reference structure and calculation of several global similarity and distance measures (Magnus et al. 2020): root mean square deviation (RMSD) (Kabsch 1978); deformation index (DI) that normalizes RMSD with the sequence length (Parisien et al. 2009); interaction network fidelity (INF), including Watson-Crick, noncanonical, and stacking interactions (Parisien et al. 2009); and, more recently, mean of circular quantities operating in torsion angle space (Zok et al. 2014; Wiedemann et al. 2017). RMSD serves as the main criterion to rank the predicted models, although it is only capable of assessing the minimum average distance between two 3D structures represented as two sets of atomic coordinates. The remaining metrics allow a focus on base pairs and torsion angles. Additionally, RNA-Puzzles uses the Clashscore-as defined in the MolProbity software package (Williams et al. 2018)_for assessing the accuracy in a noncomparative procedure by finding overlapping or too close atoms in the models and used as an overall evaluation of the stereochemistry.

Nevertheless, current biological problems are setting new thresholds of what acceptable geometry qualities should be. Catalytic features, for instance, highlight that not only the model's geometry is important, but also its stereochemistry is an important factor as well. One example is the torsion-angle-based dependence between the active and nonactive conformation of base pairs in some ribozyme active sites (White et al. 2018). Moreover, selfcleaving ribozymes can provide another example, in which the correct description of phosphate backbone stereochemistry is critical to correctly assess the reaction pathways of these mechanisms (Teplova et al. 2020). Yet another recent case is drug development that targets RNA (e.g., against viruses) (Aftab et al. 2020). Therefore, there is a clear need to advance technology to provide useful and trustable tools capable to address these challenges.

Proper stereochemistry is at the core of biomolecular structure modeling. The geometries and stereochemistry of the nucleic acid building blocks are very well known and with high precision (Clowney et al. 1996; Gelbin et al. 1996; Schneider et al. 1996). Inaccuracies in molecular geometry can result from geometry optimizations that fall into local minima that may lead to a metastable conformer, different from the native one or another biologically irrelevant conformation. Inappropriate geometry may mask incorrect choice in torsion-angle space (for example, a base incorrectly in the syn conformer can lead to geometrical distortions in the sugar-phosphate backbone). Biomolecular structures are extremely well finetuned and the whole variety of physicochemical interactions is exploited in the folded native structure. Neglect of some type of interactions, or an inappropriate calibration, can lead to wrong conformations that can produce molecular distortions under insufficiently controlled structural refinement (Popenda et al. 2021).

Here, we revisit the evaluation of the stereochemistry of predicted models beyond interatomic noncovalent distances. We follow a routine recommended to experimenters who deposit their structure data in the Protein Data Bank (Berman et al. 2000) and the Biological Magnetic Resonance Data Bank (Ulrich et al. 2008)_both contributing to the wwPDB partnership (Berman et al. 2003). wwPDB stresses the importance of careful examination of structures by providing tools that set the standards for 3D structure submission. In 2017, it introduced OneDep-a unified system applying the deposition, biocuration, and validation pipelines for structural data (Gore et al. 2017; Young et al. 2017). OneDep is an extensive suite of programs operating on different metrics to assess the accuracy of structures. It implements stereochemistry analysis through MAXIT (Feng et al. 1998; Berman et al. 2000).

To evaluate the stereochemistry of RNA tertiary structure predictions, we analyzed the results of all RNA-Puzzles challenges with the standardized data available as of November 2020-that is, puzzles 1-15, 17-21, and 24 (puzzle 14 in two versions, bound 14a and free 14b) -and 22 corresponding reference structures. We downloaded 1030 predicted RNA models from the standardized data set belonging to RNA-Puzzles resources (located at https:// github.com/RNA-Puzzles; Magnus et al. 2020). Among those, 797 models were in the human category and 233 models in the web server category. From these data, we created 23 clusters by participants-each containing models submitted by a single human group or a web server (Table 1). An additional 24th cluster included the reference structures (Table 2). We processed the structures in all of these subsets using MAXIT software (Feng et al. 1998; Berman et al. 2000) and compared results with the MolProbity software (Williams et al. 2018). Next, we used Barnaba (Bottaro et al. 2019) and X3DNA-DSSR (Lu and Olson 2003) to verify base-paring geometries and handedness of helices, respectively. Finally, we conducted a simple statistical analysis by computing the average value, standard deviation, and median for every subset including more than one model (see Materials and Methods).

\section{RESULTS}

For each model, MAXIT returned a report of abnormal stereochemical parameters (falling into six categories: close 


\section{Carrascoza et al.}

TABLE 1. Clusters of RNA 3D models predicted within RNA-Puzzles organized by participants

\begin{tabular}{|c|c|c|c|c|c|}
\hline Category & Cluster & $\begin{array}{l}\text { Name of the group/ } \\
\text { server }\end{array}$ & $\begin{array}{l}\text { Number of } \\
\text { challenges }\end{array}$ & $\begin{array}{c}\text { Total number of predicted } \\
\text { models }\end{array}$ & $\begin{array}{c}\text { Puzzle (challenge) } \\
\text { numbers }\end{array}$ \\
\hline \multirow[t]{16}{*}{$\begin{array}{l}\text { Human } \\
\text { groups }\end{array}$} & $\mathrm{H} 1$ & Adamiak & 14 & 64 & $\begin{array}{l}4,5,7,8,11-15,17 \\
19-21,24\end{array}$ \\
\hline & $\mathrm{H} 2$ & Bujnicki & 20 & 131 & $1-14,17,19-21,24$ \\
\hline & $\mathrm{H} 3$ & Chen & 20 & 145 & $1-19,21$ \\
\hline & $\mathrm{H} 4$ & Das & 21 & 188 & $1-14,17-21,24$ \\
\hline & H5 & Ding & 12 & 124 & $7-9,11-14,17-19,24$ \\
\hline & $\mathrm{H} 6$ & Dokholyan & 14 & 52 & $1-10,13,17-19$ \\
\hline & $\mathrm{H} 7$ & Kollmann & 1 & 10 & 24 \\
\hline & $\mathrm{H} 8$ & Lee & 1 & 5 & 18 \\
\hline & H9 & Major & 7 & 32 & $1-4,6-7,17$ \\
\hline & $\mathrm{H} 10$ & Mikolajczak & 1 & 1 & 4 \\
\hline & $\mathrm{H} 11$ & Sanbonmatsu & 1 & 4 & 21 \\
\hline & $\mathrm{H} 12$ & Santalucia & 3 & 3 & $1,2,4$ \\
\hline & $\mathrm{H} 13$ & Weeks & 1 & 3 & 12 \\
\hline & $\mathrm{H} 14$ & Wildauer & 1 & 1 & 2 \\
\hline & $\mathrm{H} 15$ & Xiao & 6 & 33 & $5,11-13,17,20$ \\
\hline & $\mathrm{H} 16$ & Yagoubali & 1 & 1 & 18 \\
\hline \multirow[t]{7}{*}{ Web servers } & W1 & 3dRNA & 5 & 30 & $15,18-19,21,24$ \\
\hline & W2 & FARFAR & 6 & 70 & $15,18-21,24$ \\
\hline & W3 & iFoldRNA & 1 & 5 & 24 \\
\hline & W4 & LeeServer & 2 & 10 & $18-19$ \\
\hline & W5 & RNAComposer & 7 & 49 & $15,17-21,24$ \\
\hline & W6 & SimRNA & 7 & 59 & $15,17-21,24$ \\
\hline & W7 & Vfold & 1 & 10 & 24 \\
\hline
\end{tabular}

contacts, bond length deviations, bond angle deviations, deviation from planarity, chirality issues, and phosphate bond linkages (Supplemental Material includes tables with the error numbers in every model). Using MAXIT, we examined them first for the subset of 22 reference structures (Fig. 1; Supplemental Table S1). Most of them contained some types of geometrical deviations from standard dictionaries. We found the highest incidence of errors in the bond angles (183 errors in 17 structures), followed by close contacts (54 errors in seven structures) and bond lengths (32 errors in five structures). Among the worst cases (PZ07, PZ01, and PZ21), two are for structures at a resolution worse than $2.5 \AA$ (cf. Supplemental Fig. S1 in the Supplemental Material). The software X3DNADSSR (Lu and Olson 2003) does not reveal any lefthanded helix/dinucleotide step in RNA-Puzzles submissions nor experimentally determined RNA 3D structures (cf. Supplemental Tables S46-S69 in the Supplemental Material). In Figure 1, one can also observe that there are no chirality issues, while deviations from planarity occur only in two instances (nine errors in total). For polymer linkage (i.e., deviations in $\mathrm{P}-\mathrm{O}$ bond lengths), we found seven structures with a total of nine reported inaccuracies, making an average of less than one error per structure, the same as for errors in planarity.

We have analyzed separately clusters with models predicted by human experts and web servers. Each of these
23 collections contains the predictions submitted by one participant within all the considered challenges available in the standardized data set of RNA-Puzzles resource (cf. Table 1). Their cardinalities range from 1 to 188. Within each of these clusters, except for those including only one model (i.e., $\mathrm{H} 10, \mathrm{H} 14$, and $\mathrm{H} 16$ ), we determined the total number of errors of each type (Supplemental Tables S2-S24 in the Supplemental Material), the average number over all the errors and the standard deviation (Fig. 2) and confirmed these results using MolProbity software, version 4.5.1 (Supplemental Figs. S2-S4; Supplemental Tables S71-S92 in the Supplemental Material; Williams et al. 2018). We did the same for each of the six types of stereochemical properties; we further computed the average value of each error and the standard deviation per cluster (Fig. 3). One can observe that some of the applied prediction methods have an advantage over others in terms of the total number of errors. However, most submissions have stereochemical issues to address. Interestingly, there is no visible difference between the qualities of human versus web server predictions as far as the average number of all the inaccuracies is concerned. In both categories, we can observe both good and bad scores. The average number of errors per model in the human category equals 106, while in the web server category it is 103 .

Due to the significant difference between cluster cardinalities, there is no statistical consistency between them, 
TABLE 2. Cluster with the reference structures and their prediction-related data

\begin{tabular}{|c|c|c|c|c|c|c|c|c|}
\hline \multirow[b]{2}{*}{ No. } & \multirow[b]{2}{*}{ Puzzle ID } & \multirow[b]{2}{*}{ PDB ID } & \multicolumn{3}{|c|}{ Number of predicted models } & \multicolumn{3}{|c|}{ Number of participants } \\
\hline & & & Humans & Web servers & Total & Humans & Web servers & Total \\
\hline 1 & PZ01 & $3 \mathrm{MEI}$ & 14 & 0 & 14 & 6 & 0 & 6 \\
\hline 2 & PZO2 & 3P59 & 13 & 0 & 13 & 7 & 0 & 7 \\
\hline 3 & PZO3 & $30 W Z$ & 12 & 0 & 12 & 5 & 0 & 5 \\
\hline 4 & PZO4 & 3V7E & 30 & 0 & 30 & 8 & 0 & 8 \\
\hline 5 & PZ05 & 4P9R & 25 & 0 & 25 & 7 & 0 & 7 \\
\hline 6 & PZ06 & $4 G X Y$ & 34 & 0 & 34 & 5 & 0 & 5 \\
\hline 7 & PZO7 & $4 \mathrm{R} 4 \mathrm{~V}$ & 52 & 0 & 52 & 7 & 0 & 7 \\
\hline 8 & PZ08 & 4L81 & 42 & 0 & 42 & 6 & 0 & 6 \\
\hline 9 & PZ09 & $5 K P Y$ & 34 & 0 & 34 & 5 & 0 & 5 \\
\hline 10 & PZ10 & 4LCK & 26 & 0 & 26 & 4 & 0 & 4 \\
\hline 11 & PZ11 & 5IEM & 53 & 0 & 53 & 6 & 0 & 6 \\
\hline 12 & PZ12 & 4QLM & 51 & 0 & 51 & 7 & 0 & 7 \\
\hline 13 & PZ13 & $4 \times W 7$ & 55 & 0 & 55 & 7 & 0 & 7 \\
\hline 14 & PZ14a & 5DDO & 61 & 0 & 61 & 5 & 0 & 5 \\
\hline 15 & PZ14b & 5DDO & 52 & 0 & 52 & 5 & 0 & 4 \\
\hline 16 & PZ15 & $5 \mathrm{D} \mid 4$ & 20 & 54 & 74 & 2 & 4 & 6 \\
\hline 17 & PZ17 & $5 K 7 C$ & 69 & 38 & 107 & 8 & 2 & 10 \\
\hline 18 & PZ18 & 5TPY & 24 & 28 & 52 & 6 & 5 & 11 \\
\hline 19 & PZ19 & $5 T 5 A$ & 26 & 28 & 54 & 6 & 5 & 11 \\
\hline 20 & PZ20 & $5 Y 85$ & 20 & 20 & 40 & 4 & 3 & 7 \\
\hline 21 & PZ21 & $5 N Z 6$ & 34 & 25 & 59 & 5 & 4 & 9 \\
\hline 22 & PZ24 & $6 \mathrm{OL3}$ & 50 & 40 & 90 & 5 & 6 & 11 \\
\hline
\end{tabular}

but there is statistical consistency within each cluster-the results of a single participant. For instance, the $\mathrm{H} 9$ set, for which the total score is significant in Figure 2, has only 32 items - we should remember that in a small set, one highly defective object significantly affects the average value and standard deviation. The most numerous clusters (over 100 models) are $\mathrm{H} 2, \mathrm{H} 3, \mathrm{H} 4$, and $\mathrm{H} 5$. The sets labeled as $\mathrm{H} 1$, H6, W2, and W6 include 50-100 items. The remaining ones have less than 50 models each. By clustering and comparing the predictions submitted, one can observe that $\mathrm{H} 1$ (average total number of errors, $\operatorname{ATN}=0), \mathrm{H} 4$ (ATN = 7.69), and H8 (ATN $=11.60)$ groups apply methods performing the best in the category of human experts; W2 (ATN $=1.30)$, W5 (ATN = 7.61), and W4 (ATN = 31.20) are most successful among the web servers-their average number of all errors per model is less than 50 (Fig. 2). For clusters $\mathrm{H} 2, \mathrm{H} 3, \mathrm{H} 5, \mathrm{H} 6$, $\mathrm{H} 11, \mathrm{H} 12, \mathrm{H} 13$, and $\mathrm{W} 1$, the average total number of inaccuracies is in the range of 50-200. However, the significant standard deviations indicate a large spread in stereochemical issues for the prediction methods used to obtain the models collected in these clusters. In the other clusters, the average total number of errors falls in the range of 200-350 (if we do not consider singlemodel clusters).

A comparison between Figures 1 and 2 shows the gap between reference structures and predicted models. The most notable conformational errors in predicted models

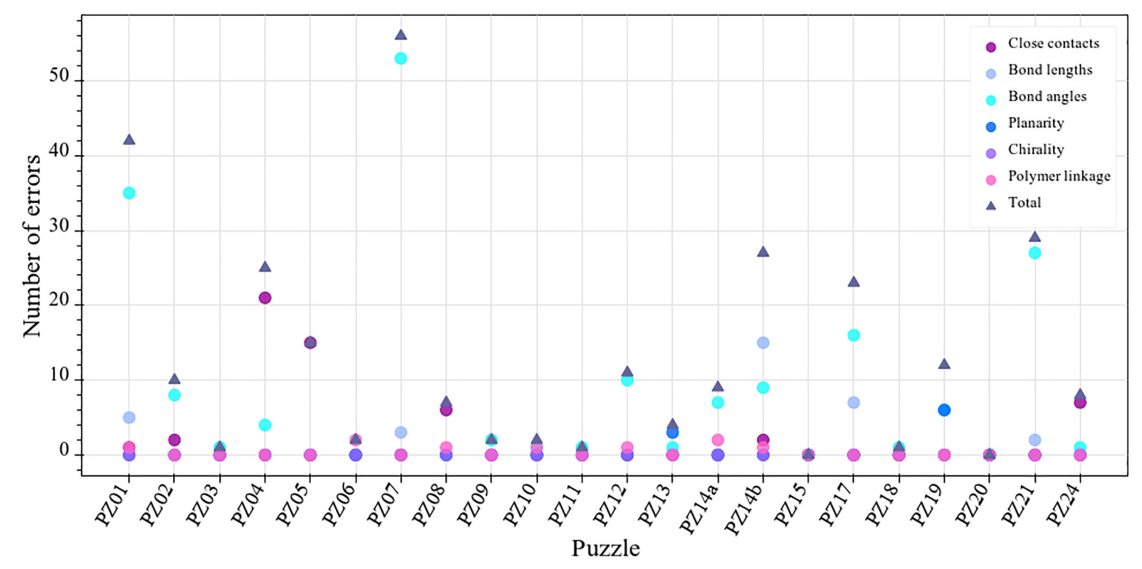

FIGURE 1. Stereochemical errors were reported by MAXIT for the reference structures. 

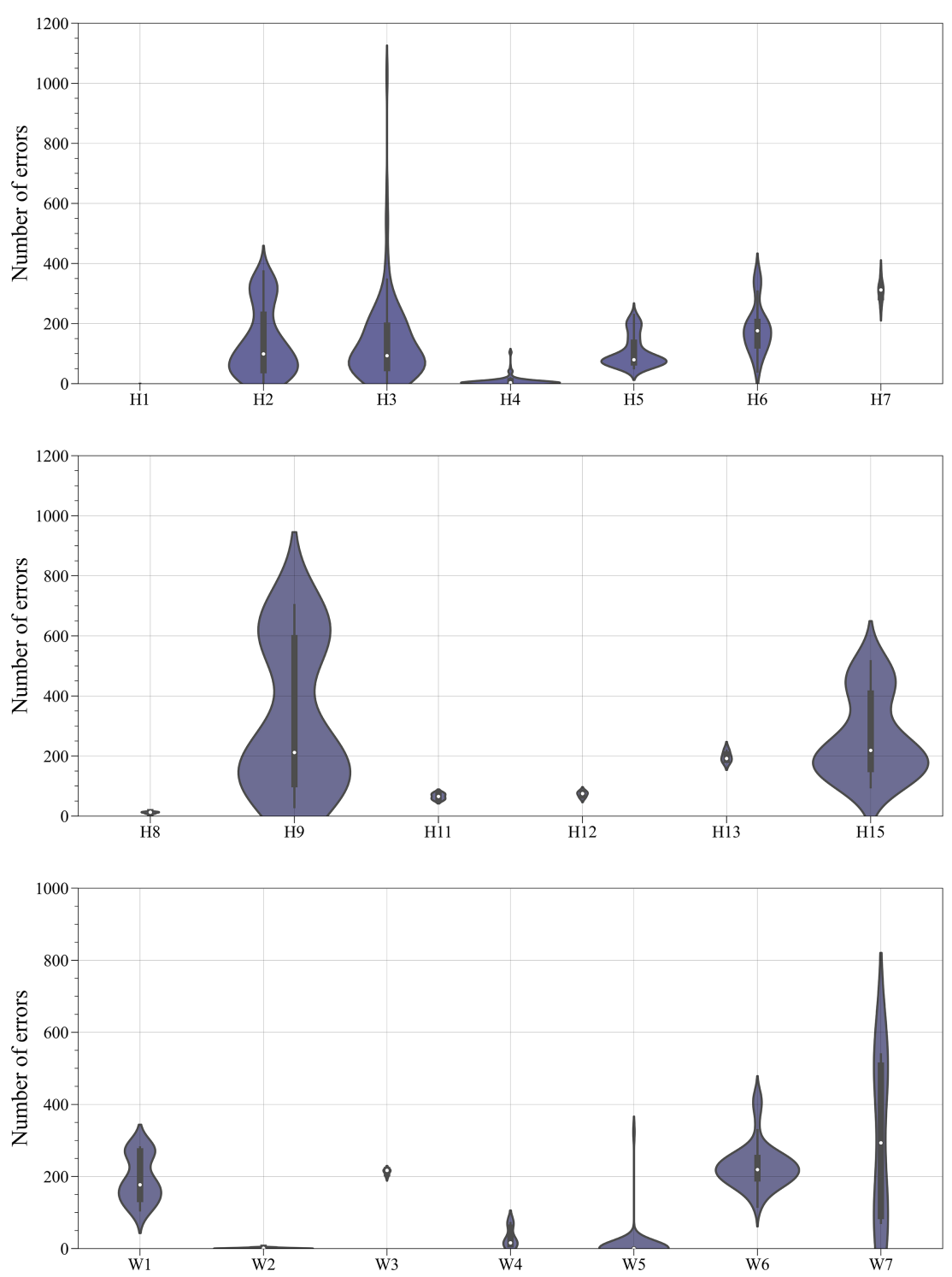

FIGURE 2. The number of stereochemical errors identified in all the considered models by participants. The white dot at the center of every violin plot represents a median. The black bar corresponds to the interquartile range. The first and the third quartile are represented as wicks up and down from the interquartile range. The violin shape shows error distribution. es of the protocols embedded within various prediction programs. The plots reveal the highest number of inaccuracies especially in bond angles $(70,184)$. Virtually every prediction method generates errors in covalent geometries, and the exceptional models with no such issue are not necessarily the most similar to the reference structure(s) in terms of overall RMSD. In the human category, models collected in $\mathrm{H} 1, \mathrm{H} 4$, and $\mathrm{H} 8$ clusters have little or no geometric issues (although, at the same time, four $\mathrm{H} 4$ models in puzzle 19 have the largest bond length error with $\mathrm{O5}^{\prime}$ -C5' length $>100 \AA$ ), while predictions in $\mathrm{H} 7, \mathrm{H} 9$, and $\mathrm{H} 15$ are among those with the highest number of inaccuracies. In the web server category, W2, W4, and W5 perform the best as far as bond lengths and angles are concerned, while W3, W6, and W7 are at the end of the ranking. If we consider deviations from ring planarity (Fig. 3), of which the total number is 17,594 , their average per model for every cluster is below 90 errors. Models in $\mathrm{H} 6, \mathrm{H} 9$, and $\mathrm{H} 13$ have a significant number of these issues. The largest identified deviation from planarity equals $0.791 \AA$ and occurred in H15 model 1 predicted for puzzle 12. An example error of this type is depicted in Supplemental Figure S5.

The average number of chirality errors is below 20 for all clusters. For some clusters, MAXIT reported zero or one issue of this type in total: $\mathrm{H} 1$, $\mathrm{H} 8$, and $\mathrm{H} 13$ within the human category, and W2, W3, and W5 within

occur in bond lengths and angles. On average, MAXIT has identified over a hundred of such inaccuracies per the predicted RNA 3D model and less than 10 per the reference structure (on average). Chirality (or incorrect sugar substituent) is correct in the experimentally determined RNAs, while $28 \%$ of the predicted models have problems with it. Quite many deviations from the average planes of aromatic rings are observed. Polymer linkage assesses bond lengths between the adjacent nucleotides by measuring the $\mathrm{P}-\mathrm{O}$ bond distances. This parameter has the lowest error rate in computationally generated structures-errors of this type occurred in $22 \%$ of all analyzed RNAs.

Figure 3 presents MAXIT results separately for each error type and allows us to take a closer look into the weakness- the web servers. Let us add that for $\mathrm{H} 4$-having the longest track of submissions-the number of errors in this category is also negligible. Some approaches $(\mathrm{H} 2, \mathrm{H} 15$, and W4) scored higher as far as the average number of chirality inaccuracies is concerned.

In total, 2130 abnormalities classified by MAXIT as chirality errors occurred in 291 predicted models. The most common form of chiral error is the interchange of the hyatom at the ribose moiety (Fig. 4). Such an interchange does not lead to a chiral error; it produces another sugar type (for example arabinose or xylose instead of ribose). Such improper sugar construction represents $94.9 \%$ of all chiral errors identified by MAXIT. The remaining 5.1\% droxyl group and hydrogen atom on the same carbon 

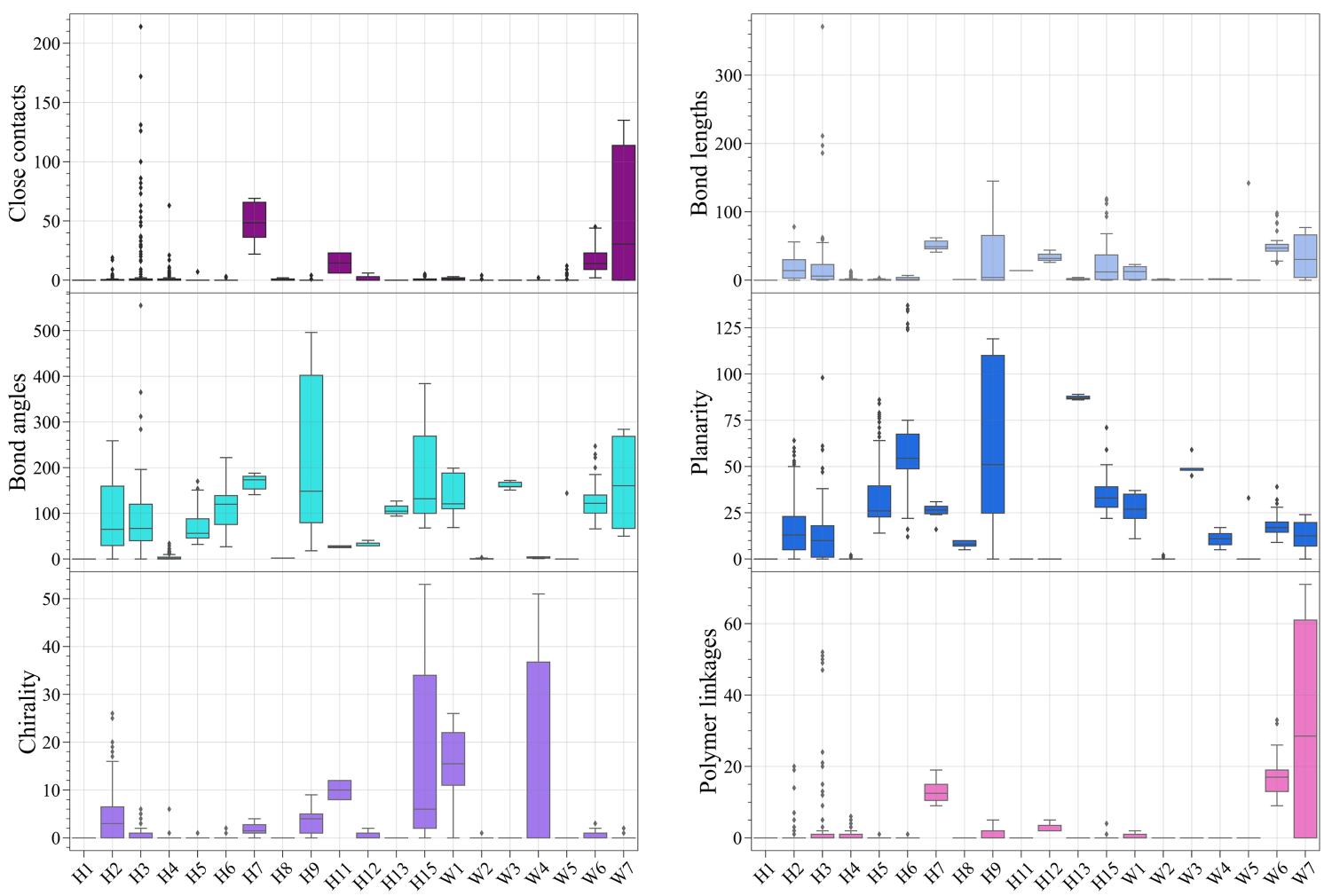

FIGURE 3. The number of identified stereochemical errors per error type and participant. The box plot of each participant shows the interquartile range. The black middle line in every box depicts a median. The first and third quartiles are represented as wicks up and down from the interquartile range. Separated dots outside boxes correspond to outliers.

are planar inaccuracies in the sugar ring, and they occur when the improper torsion angle at a $\mathrm{sp}^{3}$ carbon atom is close to zero instead of being around -122 or +122 degrees. Such a situation occurs in the furanose ring with distorted or flat sugar rings.

A distribution of chiral errors among nucleotides is shown in Table 3. We can see a high frequency in guanine (692 inaccuracies, which make $32.5 \%$ of all chiral errors) and a lower one for uracil (410 inaccuracies, which make $19.2 \%$ of all chiral errors). This relationship is visible for both sugar construction inversions and planar errors. However, the frequencies are affected by the nucleotide content in the analyzed RNA structures. Thus, in Table 3, we also present the total number of adenines, cytosines, guanines, and uracils in the analyzed data set, and the percentage of these nucleotides having erroneous chirality. Let us add that among all nucleotides with chiral errors, $91 \%$ are anti while $9 \%$ are syn nucleotides. A similar distribution is observed for each of the individual nucleotide types. Syn/anti conformation characterizes a relative orientation of base and sugar and is determined based on the $\chi$-torsion angle (defined by $\mathrm{O} 4$ '-C1'-N1-C2 for pyrimidines and $\mathrm{O}^{\prime}-\mathrm{C} 1^{\prime}-\mathrm{N} 9-\mathrm{C} 4$ for purines). Usually, $\chi$ falls into the ranges $[+90,+180]$ or $[-180,-90]$ corresponding to the anti conformation. Occasionally, we observe its value in $[-90,+90]$, which refers to the syn conformation.
Some chiral errors (11\%) appear when the conformation of a nucleotide in the predicted model differs from that in the reference structure. However, in most cases (89\%), these errors cannot result from the conformation change
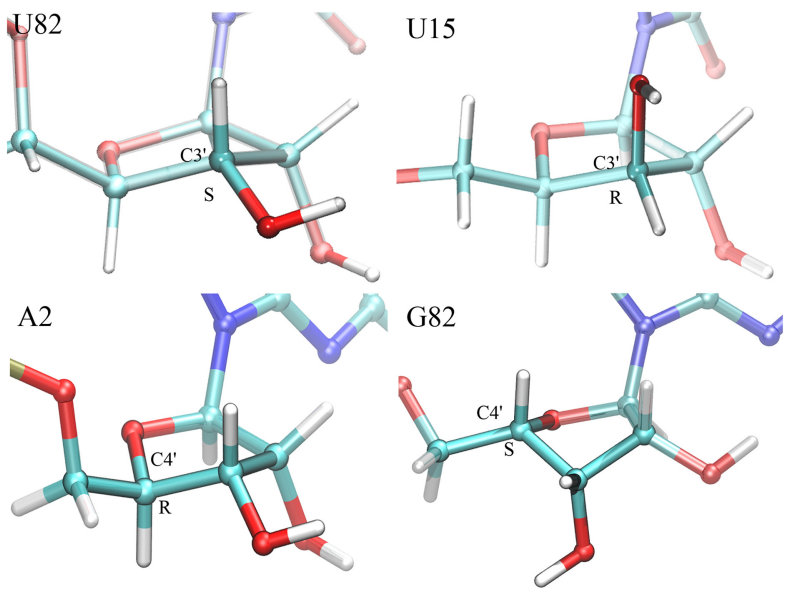

FIGURE 4. Example chiral errors in $\mathrm{H} 3$ model from $\mathrm{PZO}$ (top) and $\mathrm{H} 4$ model from PZO6 (bottom). (Top left) C3' atom in U82 with correct chiral center. (Top right) U15 with incorrect chiral inversion at carbon atom $\mathrm{C}^{\prime}$, actually changing a ribose to a xylose moiety. (Bottom left) A2 with correct chirality at C4'. (Bottom right) G82 with incorrect chirality at $\mathrm{C}^{\prime}$. 


\section{Carrascoza et al.}

TABLE 3. Chiral or planar sp3 atom errors by nucleotide type

\begin{tabular}{|c|c|c|c|c|c|c|c|}
\hline \multirow[b]{2}{*}{ Nucleotide } & \multirow{2}{*}{$\begin{array}{l}\text { Nucleotide content } \\
\text { in the data set }\end{array}$} & \multicolumn{3}{|c|}{ Number of erroneous nucleotides } & \multicolumn{3}{|c|}{ Percentage of erroneous nucleotides (\%) } \\
\hline & & Total & Reversed geometry & Planar sugar & Total & Reversed geometry & Planar sugar \\
\hline A & $1,184,711$ & 585 & 554 & 31 & 0.049 & 0.047 & 0.002 \\
\hline C & $1,198,575$ & 443 & 419 & 24 & 0.037 & 0.035 & 0.002 \\
\hline G & $1,679,526$ & 692 & 655 & 37 & 0.041 & 0.039 & 0.002 \\
\hline U & 872,633 & 410 & 393 & 17 & 0.047 & 0.045 & 0.002 \\
\hline Total & $4,935,445$ & 2130 & 2021 & 109 & 0.043 & 0.041 & 0.002 \\
\hline
\end{tabular}

The percentage of erroneous nucleotides of a given type is calculated about all nucleotides in the set of analyzed models.

(Table 4). Regarding the distribution of errors among chiral atomic centers, we can observe that $43 \%$ of inaccuracies occur at C4' (cf. Fig. 4), 25\% at C2' and C3' (cf. Fig. 4), and only $5.9 \%$ at $\mathrm{C}^{\prime}$ atom (Table 5 ).

Polymer linkage errors are rare for most predicted models (Supplemental Fig. S6). We have found that MAXIT may report false positives in this category-whenever it comes to the truncation of the sequence in the model, MAXIT fails to recognize different chains properly. This kind of artifact is clear for the reference structures (e.g., PZ12 and PZ21). However, when it comes to RNA 3D models predicted by web servers or human experts, we have not found such false positives since different chains are labeled correctly by the prediction methods ( $10 \%$ of submissions contain doublechain models). Thus, polymer linkage inaccuracies depicted in Supplemental Figure S6 are true-positive errors, and they come from incorrect linkage bond length between oxygen and phosphate group of two neighboring nucleotides in the polymer chain (Supplemental Fig. S6). Such errors were suspected to occur during the assembly building process of RNA fragments since generally nucleotides start at $5^{\prime}-\mathrm{P}$ and end at O3'; however, our analysis revealed that models predicted by assembly-based methods did not show errors of this type.

The analysis of the RNA-Puzzles data set containing 1052 RNAs reveals 2431 polymer linkage errors, including 2422 errors in 230 predicted models and nine errors in seven reference structures. $\mathrm{H} 2$ and $\mathrm{H} 7$ clusters have the highest average number of these errors among human expert predictions. For the web servers, MAXIT has found the highest number of this type of inaccuracy in W6 and W7. The remaining prediction methods do not tend to generate errors in this category. By default, MAXIT reports such error whenever the distance between oxygen atom $\mathrm{O}^{\prime}$ ' and phosphorus atom $\mathrm{P}$ of the next nucleotide in the polymer chain is longer than a typical length of a covalent bond between these atoms (Schneider et al. 1996). Some of these errors are small deviations, but major ones occur as well. For example, in $\mathrm{H} 15$ model three predicted within Puzzle 13, MAXIT identified a bond of length $82.52 \AA$ between A70 and A71-it is the highest inaccuracy of this type identified within the data set. The distribution of errors by the nucleotide type and syn/anti conformation is presented in Table 6. One can observe that linkage errors involving adenine (19\%) are least frequent, and those with guanine (32\%) occur most often. However, as a function of the relative contents of the four nucleotides in the analyzed RNA molecules, cytosine has the most linkage errors and adenine the least.

Then, we analyzed the data set with the Barnaba software (Bottaro et al. 2019), and we computed the backbone root mean square deviation (herein called BBRMSD) and base-

TABLE 4. Chiral or planar $\mathrm{sp}^{3}$ atom errors by nucleotide type and conformation (anti or syn) depending on whether it is different or the same as in the reference structure

\begin{tabular}{|c|c|c|c|c|c|c|}
\hline \multirow[b]{2}{*}{ Nucleotide } & \multicolumn{3}{|c|}{ Number of erroneous nucleotides } & \multicolumn{3}{|c|}{ Percentage of erroneous nucleotides (\%) } \\
\hline & Total & Same conformation & Changed conformation & Total & Same conformation & Changed conformation \\
\hline A & 585 & 524 & 61 & 27.46 & 24.60 & 2.86 \\
\hline C & 443 & 405 & 38 & 20.80 & 19.01 & 1.79 \\
\hline G & 692 & 623 & 69 & 32.49 & 29.25 & 3.24 \\
\hline$U$ & 410 & 339 & 71 & 19.25 & 15.92 & 3.33 \\
\hline Total & 2130 & 1891 & 239 & 100 & 88.78 & 11.22 \\
\hline
\end{tabular}

The percentage of erroneous nucleotides of a given type and conformation is calculated about all (2130) erroneous nucleotides in the set. 
TABLE 5. Chiral or planar sp3 atom errors

\begin{tabular}{|c|c|c|c|c|c|}
\hline Atom & $C 1^{\prime} \beta / \alpha$ & $\mathrm{C} 2{ }^{\prime} \mathrm{R} /$ arabino & C3' R/xylose & $C 4^{\prime} \mathrm{D} / \mathrm{L}$ & Total \\
\hline Number of errors & 163 & 521 & 589 & 857 & 2130 \\
\hline Percentage of errors (\%) & 7.65 & 24.46 & 27.65 & 40.23 & 100 \\
\hline
\end{tabular}

pairing interactions root mean square deviations (eRMSD) for nucleobases. BBRMSD can be interpreted as the quality of the backbone structures expressed in RMSD values. eRMSD was taken to measure the quality of base pairs in the structures. Both values were computed using the reference structure given by the RNA-Puzzles organizers.

eRMSD values $<8 \AA$ are considered as low. For values $<5 \AA$, both the reference structure and target structure are very similar (Bottaro et al. 2019).

Our results (see Supplemental Fig. S7A) show that across all the puzzles, there is no sensitive trend in the average measurement of both BBRSMD and eRMSD. In the average RMSD values across participants (see Supplemental Fig. S7B), groups $\mathrm{H} 1$ to $\mathrm{H} 4$ and $\mathrm{H} 12$ performed better than the automated protocols, with the exception of W7, which showed the lowest average in both, backbone and basepair quality. Some groups, from $\mathrm{H} 6$ to $\mathrm{H} 9$, have lower eRMSD than BBRMSD values, suggesting these groups focus their attention on the base pairs rather than the backbone structure. Other groups, like H11 and W5, perform better at deducing the volumetric backbone shape, but they have relatively worst base-pair performance (Supplemental Fig. S7B).

\section{DISCUSSION}

Stereochemical errors in the predicted RNA 3D models are primarily generated by computer programs used in both human-guided and web server prediction. In many cases, these may be the result of relatively small rounding errors appearing at one of the calculation steps and propagating in subsequent iterations. Knowing the general approach used by the prediction method, it is possible to indicate the most sensitive stages at which the errors arise.

Computational complexity is the main problem in de novo simulation of RNA folding. Therefore, different techniques are used to reduce the time cost of de novo prediction methods. One of them is to evaluate the fold using simplified potentials, which do not take stereochemical parameters into account. The simulation (e.g., Monte Carlo) converges toward an optimum defined in terms of the overall 3D shape of the molecule and gives a fold that is stereochemically oversimplified and far from ideal. The large number of calculations that are performed during the random sampling of the solution space also affects the generation of errors, as the errors that occur in one step are propagated further and often deteriorate the final solution. Another related problem concerns coarse-grained simulations. The transformation of a coarse-grained model to a full-atom model is a highly erroneous procedure.

In template-based approaches (homology modeling, and fragment assembly methods), a crucial moment is the choice of the right template/fragment. A model based

TABLE 6. Polymer linkage errors by the types and conformation (anti or syn) of nucleotides depending on whether they have the same or different conformation as in the reference structure

\begin{tabular}{|c|c|c|c|c|c|c|c|c|}
\hline \multirow[b]{3}{*}{ Linkage } & \multirow[b]{3}{*}{ Total } & \multicolumn{3}{|c|}{ Number of erroneous linkages } & \multirow[b]{3}{*}{ Total } & \multicolumn{3}{|c|}{ Percentage of erroneous linkages (\%) } \\
\hline & & \multicolumn{3}{|c|}{ Conformation $^{a}$} & & \multicolumn{3}{|c|}{ Conformation $^{a}$} \\
\hline & & Same & 1 changed & 2 changed & & Same & 1 changed & 2 changed \\
\hline $\mathrm{A}\left(\mathrm{O} 3^{\prime}\right)-\mathrm{P}$ & 461 & 378 & 72 & 11 & 19.03 & 15.61 & 2.97 & 0.45 \\
\hline $\mathrm{C}\left(\mathrm{O} 3^{\prime}\right)-\mathrm{P}$ & 692 & 652 & 38 & 2 & 28.57 & 26.92 & 1.58 & 0.07 \\
\hline $\mathrm{G}\left(\mathrm{O} 3^{\prime}\right)-\mathrm{P}$ & 783 & 714 & 60 & 9 & 32.33 & 29.48 & 2.48 & 0.37 \\
\hline$U\left(O 3^{\prime}\right)-P$ & 486 & 428 & 41 & 17 & 20.07 & 17.67 & 1.69 & 0.71 \\
\hline Total & 2422 & 2172 & 211 & 39 & 100 & 89.68 & 8.71 & 1.61 \\
\hline
\end{tabular}

a(Conformation "same") Both residues in the linkage have the same conformations in the predicted model and the reference structure; ( 1 changed) one of the predicted residues is in different conformation than in the reference structure; ( 2 changed) both predicted residues are in different conformation than in the reference structure.

The percentage of erroneous linkages of a given type and conformation is calculated about all (2422) erroneous linkages in the set. 
on a stereochemically erroneous template or of low-resolution may incorporate the incorrect stereochemical parameters. Stereochemical errors may also arise during nucleobase exchange, structural blocks insertion, or their assembly into a larger whole. In the latter case, the choice of the structural blocks that are rotated and translated is critical, since maneuvering a larger element is more erroneous.

Errors that arise during the structure modeling process can be avoided when applying a function that validates partial solutions based on their stereochemical parameters. However, it is very time-consuming and-in the case of de novo methods-completely unprofitable since it may cause the method not to return the solution in a reasonable time. Therefore, the best solution to the problem is to improve stereochemistry postfactum by minimizing the geometry or the energy after building the model, but bad local geometries are not easy to relieve when embedded in a large fold. At the same time, rerefining the templates used with standard dictionaries may alleviate the propagation of errors, leading to tight conformers with stereochemical errors difficult to energy minimize. In some tools, like FARFAR (Das and Baker 2007; Das et al. 2010) and RNAComposer (Popenda et al. 2012; Purzycka et al. 2014; Antczak et al. 2016), such a procedure has been implemented and successfully fulfills its role.

Erroneous bond lengths, bond angles, and planarity deviations are the most frequent errors in RNA 3D structure prediction, while incorrect sugar constructions or chirality and polymer linkage errors occur less frequently ( 10 issues per structure on average). False-positive errors, which are caused by improper identification of structural chains in multichain RNA structures, are found in the polymer linkage category of the MAXIT results. Most errors can be compensated by running energy minimization protocols -for example, CYANA (Güntert and Buchner 2015), NAMD (Phillips et al. 2020), XPLOR-NIH (Schwieters et al. 2003)_for the preliminary models or ensuring a proper stereochemistry from the early stages of prediction. One can also process the predicted RNA structures using tools-for example, RNAfitme (Zok et al. 2015; Antczak et al. 2018) or QRNAS (Stasiewicz et al. 2019)-having the potential to refine the nucleic acid structure.

\section{Conclusions}

We found that most RNA 3D structure prediction methods evaluated within RNA-Puzzles-either in human or web server category-generate models with some incorrect stereochemical parameters. Even the best models, according to the RMSD-based rankings, are not free of such errors. One could argue that one can generate easily a very precise model that is inaccurate and that precision in geometric and stereochemical parameters are of lesser importance. These geometric and stereochemical parameters are very well established and need to be implemented to be helpful in the future for modeling structures with catalytic or fine recognition properties. Thus, a similarity/distance measure assessing a model against a reference structure cannot be the only reliable indicator of the model quality and that all the predictors should ensure the stereochemical accuracy of their models before submission. We suggest that a detailed stereochemical analysis should enter regular evaluation processes for improving the accuracy of RNA-Puzzles submissions and promoting high-quality RNA 3D structure prediction.

\section{MATERIALS AND METHODS}

In this research, we used MAXIT version 10 downloaded from RCSB PDB (https://sw-tools.rcsb.org/apps/MAXIT), Barnaba 0.1.7 obtained from https://github.com/srnas/barnaba (Bottaro et al. 2019), MolProbity 4.5.1 taken from https://github.com/ rlabduke/MolProbity, (Williams et al. 2018), and X3DNA-DSSR, version 2.4 (Lu and Olson 2003). Structures were divided into 24 subsets: one subset with the reference structures and 23 subsets with predicted models (one for each participant), and the average values and standard deviations were computed for them. Three clusters, $\mathrm{H} 10, \mathrm{H} 14$, and $\mathrm{H} 16$, including predictions by human groups, were excluded from the statistical analysis since, in all the challenges, these groups submitted only one model each. However, their MAXIT reports are also available in the Supplemental Material.

MAXIT reports the following stereochemical issues: (1) close contacts; (2) bond length deviations; (3) bond angle deviations; (4) deviations from planarity; (5) chirality errors; and (6) polymer linkage errors (the P-O bond lengths). For 1-3 and 6, the program identifies abnormality if the parameter exceeds the expected value six times the standard $\sigma$ value. The expected values and attached $\sigma$ 's are based on Clowney et al. (1996), Gelbin et al. (1996), Parkinson et al. (1996), and Schneider et al. (1996) and are in the Supplemental Material. The current source of these reference terms is the Cambridge Structural Database (Urzhumtseva et al. 2009; Tickle 2012; Bruno and Groom 2014). Atomic clashes are signaled whenever any intermolecular atom pair is closer than the sum of their respective van der Waals radii. In general, a clash is defined when the distance between two atoms is $<2.2 \AA$ (if no $\mathrm{H}$ atom is involved) or $1.6 \AA$ (if one $\mathrm{H}$ atom is involved) (cf. Supplemental Table D1 in the Supplemental Material).

Departures from the average best-fit plane center yield the RMS deviations for all atoms from the plane. It is reported when $>6 \times 0.02 \AA$ or when at least one atom has a deviation $>0.02 \AA$. MAXIT also determines improper torsion angles in the furanose ring and reports deviations from puckering in the ring (imposed by the $\mathrm{sp}^{3}$ carbon atoms).

In the case of chirality assessment, MAXIT lists the residues that contain unexpected configuration of chiral centers (C1', C2', C3',

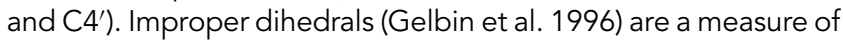
the chirality/planarity of the structure at a specific atom. 
Polymer linkage between the adjacent nucleotides is measured based on the distances computed for $\mathrm{O}^{\prime}-\mathrm{P}$ and $\mathrm{O} 5^{\prime}-\mathrm{P}$ atom pairs. By default, the $\mathrm{O} 3^{\prime}-\mathrm{P}$ distance is evaluated. However, if it exceeds $2.5 \AA$, MAXIT takes the minimum value out of these two for consideration.

Figures 4-6 were prepared using Symmetry Tool Plug-in $1.3 \mathrm{im}$ plemented in VMD software, version 1.94 (Humphrey et al. 1996).

\section{SUPPLEMENTAL MATERIAL}

Supplemental material is available for this article.

\section{ACKNOWLEDGMENTS}

This work was supported by the Polish National Science Center (grants 2016/23/B/ST6/03931 and 2019/35/B/ST6/03074 to M.S.), grant POIR.04.02.00-30-A004/16, as well as the statutory funds of Poznan University of Technology (SBAD), Poland, and the Institute of Bioorganic Chemistry, Polish Academy of Sciences.

Author contributions: F.C., M.A., and M.S. conceived the project. M.S. supervised the analysis made by F.C. and M.A. Z.M. and E.W. analyzed and assessed the results. All authors contributed to the manuscript preparation.

Received January 16, 2021; accepted November 5, 2021.

\section{REFERENCES}

Aftab SO, Ghouri MZ, Masood MU, Haider Z, Khan Z, Ahmad A, Munawar N. 2020. Analysis of SARS-CoV-2 RNA-dependent RNA polymerase as a potential therapeutic drug target using a computational approach. J Transl Med 18: 1-15. doi:10.1186/ s12967-020-02439-0

Antczak M, Popenda M, Zok T, Sarzynska J, Ratajczak T, Tomczyk K, Adamiak RW, Szachniuk M. 2016. New functionality of RNAComposer: an application to shape the axis of miR160 precursor structure. Acta Biochim Pol 63: 737-744. doi:10.18388/abp .2016_1329

Antczak M, Zok T, Osowiecki M, Popenda M, Adamiak RW, Szachniuk M. 2018. RNAfitme: a web server for modeling nucleobase and nucleoside residue conformation in fixed-backbone RNA structures. BMC Bioinformatics 19: 304. doi:10.1186/s12859-0182317-9

Berman HM, Westbrook J, Feng Z, Gilliland G, Bhat TN, Weissig H, Shindyalov IN, Bourne PE. 2000. The Protein Data Bank. Nucleic Acids Res 28: 235-242. doi:10.1093/nar/28.1.235

Berman H, Henrick K, Nakamura H. 2003. Announcing the worldwide protein data bank. Nat Struct Biol 10: 980. doi: 10.1038/nsb1203980

Boniecki MJ, Lach G, Dawson WK, Tomala K, Lukasz P, Soltysinski T, Rother KM, Bujnicki JM. 2016. SimRNA: a coarse-grained method for RNA folding simulations and 3D structure prediction. Nucleic Acids Res 44: e63. doi:10.1093/nar/gkv1479

Bottaro S, Bussi G, Pinamonti G, Reißer S, Boomsma W, LindorffLarsen K. 2019. Barnaba: software for analysis of nucleic acid structures and trajectories. RNA 25: 219-231. doi:10.1261/rna.067678 .118

Bruno IJ, Groom CR. 2014. A crystallographic perspective on sharing data and knowledge. J Comput Aided Mol Des 28: 1015-1022. doi:10.1007/s10822-014-9780-9
Clowney L, Jain SC, Srinivasan AR, Westbrook J, Olson WK, Berman HM. 1996. Geometric parameters in nucleic acids: nitrogenous bases. J Am Chem Soc 118: 509-518. doi:10.1021/ ja952883d

Cruz JA, Blanchet MF, Boniecki M, Bujnicki JM, Chen SJ, Cao S, Das R, Ding F, Dokholyan NV, Flores SC, et al. 2012. RNA-Puzzles: a CASP-like evaluation of RNA three-dimensional structure prediction. RNA 18: 610-625. doi:10.1261/rna.031054.111

Das R, Baker D. 2007. Automated de novo prediction of native-like RNA tertiary structures. Proc Natl Acad Sci 104: 14664-14669. doi:10.1073/pnas.0703836104

Das R, Karanicolas J, Baker D. 2010. Atomic accuracy in predicting and designing noncanonical RNA structure. Nat Methods 7: 291-294. doi:10.1038/nmeth.1433

Ding F, Sharma S, Chalasani P, Demidov W, Broude NE, Dokholyan NV. 2008. Ab initio RNA folding by discrete molecular dynamics: from structure prediction to folding mechanisms. RNA 14: 1164-1173. doi:10.1261/rna.894608

Feng Z, Hsieh S, Gelbin A, Westbrook J. 1998. MAXIT: macromolecular exchange and input tool. Rutgers University, New Brunswick.

Gelbin A, Schneider B, Clowney L, Hsieh S-H, Olson WK, Berman HM. 1996. Geometric parameters in nucleic acids: sugar and phosphate constituents. J Am Chem Soc 118: 519-529. doi:10.1021/ ja9528846

Gore S, Sanz García E, Hendrickx PMS, Gutmanas A, Westbrook JD, Yang H, Feng Z, Baskaran K, Berrisford JM, Hudson BP, et al. 2017. Validation of structures in the protein data bank. Structure 25: 1916-1927. doi:10.1016/j.str.2017.10.009

Gumna J, Zok T, Figurski K, Pachulska-Wieczorek K, Szachniuk M. 2020. RNAthor - fast, accurate normalization, visualization and statistical analysis of RNA probing data resolved by capillary electrophoresis. PLoS One 15: e0239287. doi: 10.1371/journal.pone .0239287

Güntert P, Buchner L. 2015. Combined automated NOE assignment and structure calculation with CYANA. J Biomol NMR 62: 453471. doi:10.1007/s10858-015-9924-9

Humphrey W, Dalke A, Schulten K. 1996. VMD: visual molecular dynamics. J Mol Graph 14: 33-38. doi:10.1016/0263-7855(96) 00018-5

Jonikas MA, Radmer RJ, Laederach A, Das R, Pearlman S, Herschlag D, Altman RB. 2009. Coarse-grained modeling of large RNA molecules with knowledge-based potentials and structural filters. RNA 15: 189-199. doi:10.1261/rna.1270809

Jossinet F, Westhof E. 2005. Sequence to structure (S2S): display, manipulate and interconnect RNA data from sequence to structure. Bioinformatics 21: 3320-3332. doi:10.1093/bioinformatics/ bti504

Kabsch W. 1978. A discussion of the solution for the best rotation to relate two sets of vectors. Acta Crystallogr A 34: 827-828. doi:10.1107/S0567739478001680

Li B, Cao Y, Westhof E, Miao Z. 2020. Advances in RNA 3D structure modeling using experimental data. Front Genet 11: 1147. doi:10 $.3389 /$ fgene. 2020.574485

Lu XJ, Olson WK. 2003. 3DNA: a software package for the analysis, rebuilding and visualization of three-dimensional nucleic acid structures. Nucleic Acids Res 31: 5108-5121. doi:10.1093/nar/ gkg680

Lukasiak P, Antczak M, Ratajczak T, Szachniuk M, Popenda M, Adamiak RW, Blazewicz J. 2015. RNAssess: a web server for quality assessment of RNA 3D structures. Nucleic Acids Res 43: W502W506. doi:10.1093/nar/gkv557

Magnus M, Antczak M, Zok T, Wiedemann J, Lukasiak P, Cao Y, Bujnicki JM, Westhof E, Szachniuk M, Miao Z. 2020. RNA-Puzzles toolkit: a computational resource of RNA 3D structure benchmark 
datasets, structure manipulation, and evaluation tools. Nucleic Acids Res 48: 576-588. doi:10.1093/nar/gkz1108

Miao Z, Westhof E. 2017. RNA structure: advances and assessment of 3D structure prediction. Annu Rev Biophys 46: 483-503. doi:10 .1146/annurev-biophys-070816-034125

Miao Z, Adamiak RW, Blanchet M-F, Boniecki M, Bujnicki JM, Chen SJ, Cheng C, Chojnowski G, Chou F-C, Cordero P, et al. 2015. RNAPuzzles Round II: assessment of RNA structure prediction programs applied to three large RNA structures. RNA 21: 10661084. doi:10.1261/rna.049502.114

Miao Z, Adamiak RW, Antczak M, Batey RT, Becka AJ, Biesiada M, Boniecki MJ, Bujnicki JM, Chen SJ, Cheng CY, et al. 2017. RNAPuzzles Round III: 3D RNA structure prediction of five riboswitches and one ribozyme. RNA 23: 655-672. doi:10.1261/rna.060368 .116

Miao Z, Adamiak RW, Antczak M, Boniecki MJ, Bujnicki JM, Chen S-J, Cheng CY, Cheng Y, Chou F-C, Das R, et al. 2020. RNA-Puzzles Round IV: 3D structure predictions of four ribozymes and two aptamers. RNA 26: 982-995. doi:10.1261/rna.075341.120

Parisien M, Major F. 2008. The MC-Fold and MC-Sym pipeline infers RNA structure from sequence data. Nature 452: 51-55. doi:10 $.1038 /$ nature06684

Parisien M, Cruz JA, Westhof E, Major F. 2009. New metrics for comparing and assessing discrepancies between RNA 3D structures and models. RNA 15: 1875-1885. doi:10.1261/rna.1700409

Parkinson G, Vojtechovsky J, Clowney L, Brünger AT, Berman HM. 1996. New parameters for the refinement of nucleic acid-containing structures. Acta Crystallogr D Biol Crystallogr 52: 57-64. doi:10.1107/S0907444995011115

Phillips JC, Hardy DJ, Maia JDC, Stone JE, Ribeiro JV, Bernardi JC, Buch R, Fiorin G, Henin J, Jiang W, et al. 2020. Scalable molecular dynamics on CPU and GPU architectures with NAMD. J Chem Phys 153: 044130. doi:10.1063/5.0014475

Popenda M, Szachniuk M, Antczak M, Purzycka KJ, Lukasiak P, Bartol N, Blazewicz J, Adamiak RW. 2012. Automated 3D structure composition for large RNAs. Nucleic Acids Res 40: e112. doi:10 $.1093 /$ nar/gks339

Popenda M, Zok T, Sarzynska J, Korpeta A, Adamiak RW, Antczak M, Szachniuk M. 2021. Entanglements of structure elements revealed in RNA 3D models. Nucleic Acids Res 49: 9625-9632. doi:10 $.1093 /$ nar/gkab716

Purzycka KJ, Popenda M, Szachniuk M, Antczak M, Lukasiak P, Blazewicz J, Adamiak RW. 2014. Automated 3D RNA structure prediction using the RNAComposer method for riboswitches. Methods Enzymol 553: 3-34. doi: 10.1016/bs.mie.2014.10.050

Rother M, Rother K, Puton T, Bujnicki JM. 2011. ModeRNA: a tool for comparative modeling of RNA 3D structure. Nucleic Acids Res 39: 4007-4022. doi:10.1093/nar/gkq1320

Schneider B, Kabelac M, Hobza P. 1996. Geometry of the phosphate group and its interactions with metal cations in crystals and ab initio calculations. J Am Chem Soc 118: 12207-12217.
Schwieters CD, Kuszewski JJ, Tjandra N, Clore GM. 2003. The Xplor$\mathrm{NIH}$ NMR molecular structure determination package. J Magn Res 160: 66-74. doi:10.1016/s1090-7807(02)00014-9

Stasiewicz J, Mukherjee S, Nithin C, Bujnicki JM. 2019. QRNAS: software tool for refinement of nucleic acid structures. BMC Struct Biol 19: 5. doi: 10.1186/s12900-019-0103-1

Teplova M, Falschlunger C, Krasheninina O, Egger M, Ren A, Patel DJ, Micura R. 2020. Crucial roles of two hydrated $\mathrm{Mg}^{2+}$ ions in reaction catalysis of the pistol ribozyme. Angew. Chem Int Ed Engl 132: 2859-2865. doi: 10.1002/ange.201912522

Tickle IJ. 2012. Statistical quality indicators for electron-density maps. Acta Crystallogr D Biol Crystallogr 68: 454-467. doi:10.1107/ S0907444911035918

Ulrich EL, Akutsu H, Doreleijers JF, Harano Y, loannidis YE, Lin J, Livny M, Mading S, Maziuk D, Miller Z, et al. 2008. BioMagResBank. Nucleic Acids Res 36: D402-D408. doi:10 $.1093 /$ nar/gkm957

Urzhumtseva L, Afonine PV, Adams PD, Urzhumtsev A. 2009. Crystallographic model quality at a glance. Acta Crystallogr D Biol Crystallogr 65: 297-300. doi:10.1107/S0907444908044296

White NA, Sumita M, Marquez VE, Hoogstraten CG. 2018. Coupling between conformational dynamics and catalytic function at the active site of the lead-dependent ribozyme. RNA 24: 1542-1554. doi:10.1261/rna.067579.118

Wiedemann J, Zok T, Milostan M, Szachniuk M. 2017. LCS-TA to identify similar fragments in RNA 3D structures. BMC Bioinformatics 18: 456. doi:10.1186/s12859-017-1867-6

Williams CJ, Headd JJ, Moriarty NW, Prisant MG, Videau LL, Deis LN, Verma V, Keedy DA, Hintze BJ, Chen VB, et al. 2018. MolProbity: more and better reference data for improved all-atom structure validation. Protein Sci 27: 293-315. doi:10.1002/pro.3330

Xu X, Zhao P, Chen S-J. 2014. Vfold: a web server for RNA structure and folding thermodynamics prediction. PLoS One 9: e107504. doi:10.1371/journal.pone.0107504

Young JY, Westbrook JD, Feng Z, Sala R, Peisach E, Oldfield TJ, Sen S, Gutmanas A, Armstrong DR, Berrisford JM, et al. 2017. OneDep: unified wwPDB system for deposition, biocuration, and validation of macromolecular structures in the PDB archive. Structure 25: 536-545. doi:10.1016/j.str.2017.01.004

Zhao Y, Huang Y, Gong Z, Wang Y, Man J, Xiao Y. 2012. Automated and fast building of three-dimensional RNA structures. Sci Rep 2: 734. doi:10.1038/srep00734

Zok T, Popenda M, Szachniuk M. 2014. MCQ4Structures to compute similarity of molecule structures. Cent Eur J Oper Res 22: 457-473. doi:10.1007/s10100-013-0296-5

Zok T, Antczak M, Riedel M, Nebel D, Villmann T, Lukasiak P, Blazewicz J, Szachniuk M. 2015. Building the library of RNA 3D nucleotide conformations using clustering approach. Int J Appl Math Comput Sci 25: 689-700. doi:10.1515/amcs2015-0050 


\section{MEET THE FIRST AUTHORS}

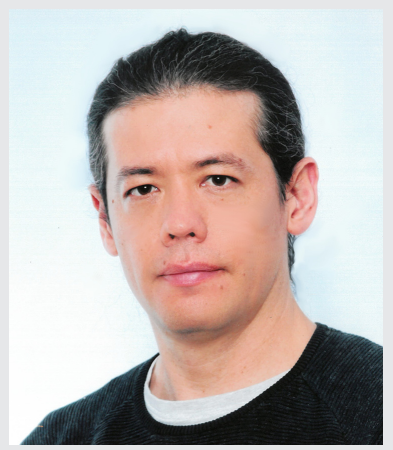

Francisco Carrascoza

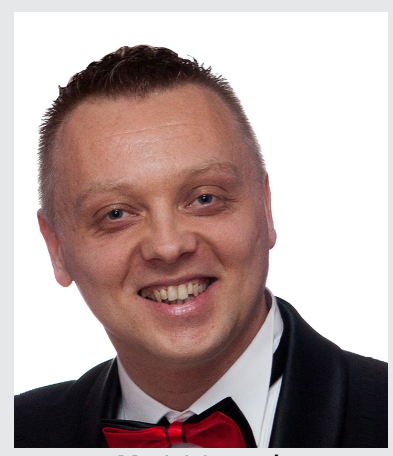

Maciej Antczak

Meet the First Author(s) is a new editorial feature within RNA, in which the first author(s) of research-based papers in each issue have the opportunity to introduce themselves and their work to readers of RNA and the RNA research community. Francisco Carrascoza and Maciej Antczak are the co-first authors of this paper, "Evaluation of the stereochemical quality of predicted RNA 3D models in the RNA-Puzzles submissions." Francisco received his $\mathrm{PhD}$ in molecular modeling from BabesBolyai University in Romania in 2016 and has been an Assistant Researcher at Poznan University of Technology since 2015, working in theoretical chemistry on ab initio molecular dynamics and metadynamics, and on the origins of life to investigate the natural formation of nucleobases and amino acids in the prebiotic world. Maciej is an Assistant Professor at the Institute of Computing Science, Poznan University of Technology, as well as the Institute of Bioorganic Chemistry, Polish Academy of Science, working on algorithms and computational methods for the analysis and prediction of RNA structures, combinatorial optimization methods for solving biologically inspired problems, high-performance computing, and artificial intelligence.

What are the major results described in your paper and how do they impact this branch of the field?

We summarized a stereochemistry-oriented evaluation of in silico RNA 3D predictions submitted for past modeling rounds of the RNA-Puzzles international contest. Most state-of-the-art methods for RNA 3D structure prediction should pay more attention to ensuring proper stereochemical features. We showed the distribution of common errors and their total count in time to outline the importance of the problem. Moreover, we quoted expected value ranges for bond lengths, torsion angles, etc., and proposed how these issues could be eliminated. We believe that our results will contribute to the improvement of RNA 3D structure prediction methods.

\section{What led you to study RNA or this aspect of RNA science?}

FC: Since my experience comes from the ab initio theories and full-atomistic level, I found that the stereochemical errors are

a challenge for those methods where the close atom-atom interactions are of higher importance than the volumetric quality of the model. We observed from our results that at full-atom scale, there is a considerable quantity of errors to mitigate, for those working on developing tools for RNA 3D structure prediction.

MA: RNAs are fascinating molecules, especially for computing scientists. Knowledge about RNA 3D folds is crucial for designing new drugs and therapeutic solutions. RNA 3D structure determination is usually expensive and not always possible. So, experimentally determined 3D structures of many biologically relevant RNAs are still unknown. The only way to mitigate this gap is an application of state-of-the-art methods for RNA 3D structure prediction. The aim of the RNA-Puzzles initiative is to stimulate the community to make efforts on continuous improvement of these methods and indirectly the quality and accuracy of RNA 3D predictions. Moreover, we give experimentalists useful hints on the successfulness of considered methods in various applications.

During the course of these experiments, were there any surprising results or particular difficulties that altered your thinking and subsequent focus?

FC: The most surprising result for me was the stable trend of the number of identified errors across all RNA-Puzzles challenges released in recent years. Therefore, we expect this report to be fruitful for the community.

MA: Surprisingly, in most of RNA-Puzzles submissions, even topranking ones, stereochemical errors have been identified. That is the reason why basic stereochemical validation could be valuable during the submission of RNA 3D predictions.

In the case of difficulties, it is not always possible to execute a bioinformatical tool cloned directly from the GitHub repository without any manual intervention. Definitely, as bioinformaticians, we need to work on this.

If you were able to give one piece of advice to your younger self, what would that be?

FC: Passion for your work will bring success. Don't worry about the things you don't have, just allow your passion to blossom.

MA: Do not be afraid of asking questions and striving persistently for answers.

What are your subsequent near- or long-term career plans?

FC: This work allowed me to realize the current struggles of the RNA community. In the near term, I plan to study in deeper detail the folding and dynamics of RNAs.

MA: I want to do what I like the most, so I plan to continue my scientific research in structural bioinformatics of RNAs. Of course, I am open to international collaboration, which could result in interesting scientific projects soon. 


\section{Carrascoza et al.}

What were the strongest aspects of your collaboration as co-first authors?

FC: The multidisciplinary exchange needed in this work between bioinformatician and chemist gave us a unique insight into different ways of approaching RNA structure. This multidisciplinary exchange, when joined, allowed us to address the problem at different levels.
MA: We represent two different scientific domains. So, our collaboration relied on the synergy of our complementary knowledge and experience. We discussed a lot but did not come across any problems that we could not overcome together. 

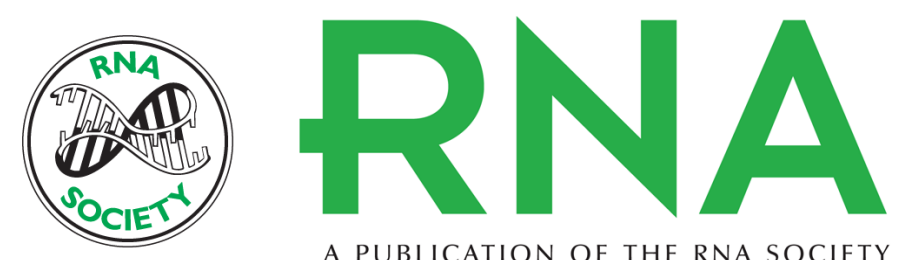

A PUBLICATION OF THE RNA SOCIETY

\section{Evaluation of the stereochemical quality of predicted RNA 3D models in the RNA-Puzzles submissions}

Francisco Carrascoza, Maciej Antczak, Zhichao Miao, et al.

RNA 2022 28: 250-262 originally published online November 24, 2021

Access the most recent version at doi:10.1261/rna.078685.121

\section{Supplemental http://rnajournal.cshlp.org/content/suppl/2021/11/24/rna.078685.121.DC1 \\ Material}

References This article cites 52 articles, 10 of which can be accessed free at: http://rnajournal.cshlp.org/content/28/2/250.full.html\#ref-list-1

Open Access Freely available online through the RNA Open Access option.

Creative This article, published in $R N A$, is available under a Creative Commons License Commons (Attribution-NonCommercial 4.0 International), as described at License http://creativecommons.org/licenses/by-nc/4.0/.

Email Alerting Receive free email alerts when new articles cite this article - sign up in the box at the Service top right corner of the article or click here.

To subscribe to $R N A$ go to:

http://rnajournal.cshlp.org/subscriptions 